\title{
FIGO Stage IB
}

National Cancer Institute

\section{Source}

National Cancer Institute. FIGO Stage IB. NCI Thesaurus. Code C96248.

A FIGO stage term that applies to gynecologic cancers. For cervical cancer, it refers to invasive cancer that is clinically visible and is confined to the cervix or it is diagnosed microscopically and the stromal invasion is more than $5.00 \mathrm{~mm}$ and the horizontal spread is more than $7.00 \mathrm{~mm}$; for endometrial cancer, it refers to cancer that is confined to the uterine body and invades one-half or more of the myometrium. 